\title{
Galactic magnetic fields in the context of dark matter. Influence on the rotation curves of spiral galaxies.
}

\author{
Joanna Jałocha \\ Institute of Physics, Cracow University of Technology, ul. Podchorążych 1, PL-30084 Kraków, \\ Poland \\ E-mail: joanna.jalocha@pk.edu.pl \\ Łukasz Bratek* \\ Institute of Physics, Cracow University of Technology, ul. Podchorazżych 1, PL-30084 Kraków, \\ Poland \\ E-mail: lukasz.bratek@pk.edu.pl
}

The large-scale magnetic fields are of paramount importance for the cosmic ray astrophysics. Patterns in the motion of interstellar medium may provide some constraints on the structure of galactic magnetic fields, especially in our Galaxy. It seems that magnetic fields cannot be ignored in attempts to fully address the problem of the rotation of spiral galaxies. The interstellar gas is ionized to a degree sufficient for the magnetic field to freeze-in the gas, so that the resulting magnetic tension influences its motion, modifying the predictions of purely gravitational models of galactic rotation. The inclusion of magnetic fields has implications for the predicted local mass-to-light ratio and so might help to reduce the missing mass problem.

36th International Cosmic Ray Conference -ICRC2019-

July 24th - August 1st, 2019

Madison, WI, U.S.A.

${ }^{*}$ Speaker. 


\section{Introduction}

The role of magnetic fields in astrophysics cannot be overestimated. To give a few examples, the fields alter the trajectories of cosmic rays, they are crucial for the physics of accretion disks, jets, neutron star magneto-spheres, for star formation processes, as well as for stellar winds. Magnetic fields cannot be ignored also when one attempts to fully understand the problem of rotation of spiral galaxies.

The turbulent and large-scale magnetic fields can influence the motion of at least partially ionized gaseous fraction. The mechanism of the influence was described in [1]. The interstellar gas is ionized to a degree sufficient for the magnetic field to freeze-in the gas, so that the resulting magnetic tension can be assumed to hold the gas and so influence its motion, modifying the predictions of purely gravitational models. Because the gas density decreases with the galactocentric distance, the magnetic effect becomes important for larger radii. Rotation curves of spiral galaxies are obtained mainly based on the observations of gaseous clouds, and these measurements are the only available in the outer regions of these galaxies. As so, the observed flatness of outer disk rotation curves, or even their rise, could be simply the result of interaction with interstellar magnetic fields. In particular, it might turn out, that taking magnetic fields into account would reduce the amount of non-baryonic dark matter required by models which ignore magnetic fields.

It should be noted that the studies on the influence of magnetic fields on rotation curves have been inconclusive so far. Some authors, e.g. [2,3], share the opinion that magnetic fields are responsible for the flattening or rise of the outer parts of rotation curves, others provide sound arguments that the influence is unlikely or even may impede the gravitationally supported rotation, leading to an even higher halo mass [4,5]. However, the effects are subject to modelling assumptions.

\section{Magnetic fields in modelling rotation curves of spiral galaxies}

A possible effect of the influence of magnetic fields on the dynamics of galaxies we described in [6] where we reported on the results concerning spiral galaxy NGC 891. The input data consisted of the rotation curve, surface brightness profile and the neutral hydrogen distribution. In the framework of global disk model, well approximating a flattened mass distribution when one disregards for a while the hypothetical dark halo, we obtained a surface mass density distribution, which in conjunction with the brighntness profile, led us to a local mass-to-light ratio profile $(M / L)$ as shown in Figure 1. This ratio, although low (1-2 in the interior), steeply increases, reaching a value 8 in the outer parts. Such a rapid increase is usually attributed to a non-baryonic dark matter halo gravitationally dominating the luminous stuff in the outer parts. But in the disk model framework, even minute changes within measurement errors in the external parts of rotation curves, may result in a significant reduction of the surface mass density. This in turn reduces the local mass-to-light profile in the outer parts. At this stage we supposed that magnetic field was responsible for the increase of the rotation curve. It turned out that the dynamical effect of magnetic fields of several micro-gauss, such as observed in NGC 891 (which is a value typical for large-scale magnetic fields in spiral galaxies $[9,10,11])$, would be sufficient to reduce the local-mass-to light ratio to acceptable lower values. 


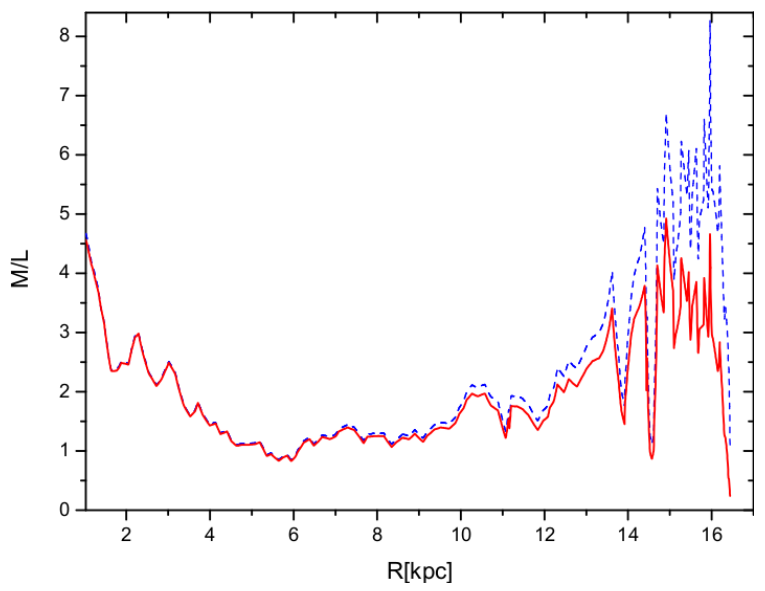

Figure 1: A change in the local mass-to-light ratio profile of spiral galaxy NGC 891 resulting from a small modification of its outer rotation curve. The original local mass-to-light ratio profile [dotted blue line] and that after accounting for the presence of magnetic field [solid red line] (only N-E quadrant considered).

The galaxy NGC 891 shows also some anomaly in the behaviour of the vertical gradient of the azimuthal component of gas velocity. This gradient, being high in the N-E quadrant, vanishes in the S-W quadrant. In this galaxy, the large-scale magnetic field is measured both inside the disk and above it. At the same time, the measurements $[9,11]$ show that the magnetic field is non-symmetric, being intense and strongly ordered in the S-W quadrant. The asymmetry in the magnetic field might be the reason of the observed asymmetry in the velocity gradient.

In [12] we investigated a possible influence of magnetic fields on the dynamics of galaxy NGC 253. The galaxy is characterized by exceptionally strong magnetic fields from 7 to 18 microgauss in the outer regions [13] (the fields may be even stronger in the central regions). The present measurements of ionised gas clouds cover more distant regions than previous rotation curve measurements [14]. The new measurements confirmed that the rotation curve of NGC 253 decreases at large distances from the centre. This indicates that the amount of dark matter may be lower than expected. Moreover, considering that the rotation curve measurements concern the ionized gas fraction, one cannot exclude an additional reduction of the expected mass-to-light profile due to interaction with magnetic fields, the more that the fields are exceptionally strong in NGC 253. As we have shown in [12], the reducing effect will be noticeable for magnetic fields of 11 microgauss, which is the value observed in this galaxy. The results are illustrated in Figure 2 and shortly discussed in the figure caption.

The main message of this observation is that one can significantly reduce the mass-to-light ratio profile in the outer regions by including magnetic fields in the disk model model. This reducing effect is easier to achieve in the disk model due to high sensitivity of the predicted mass density to small changes in the rotation curve.

\section{Magnetic field estimates from galactic rotation. The Milky Way galaxy.}

It is known that the rotation curve indicated by the cepheids and carbon stars in the outer disk of the Galaxy is markedly lower than the rotation curve of rarefied ionised gas clouds in the 

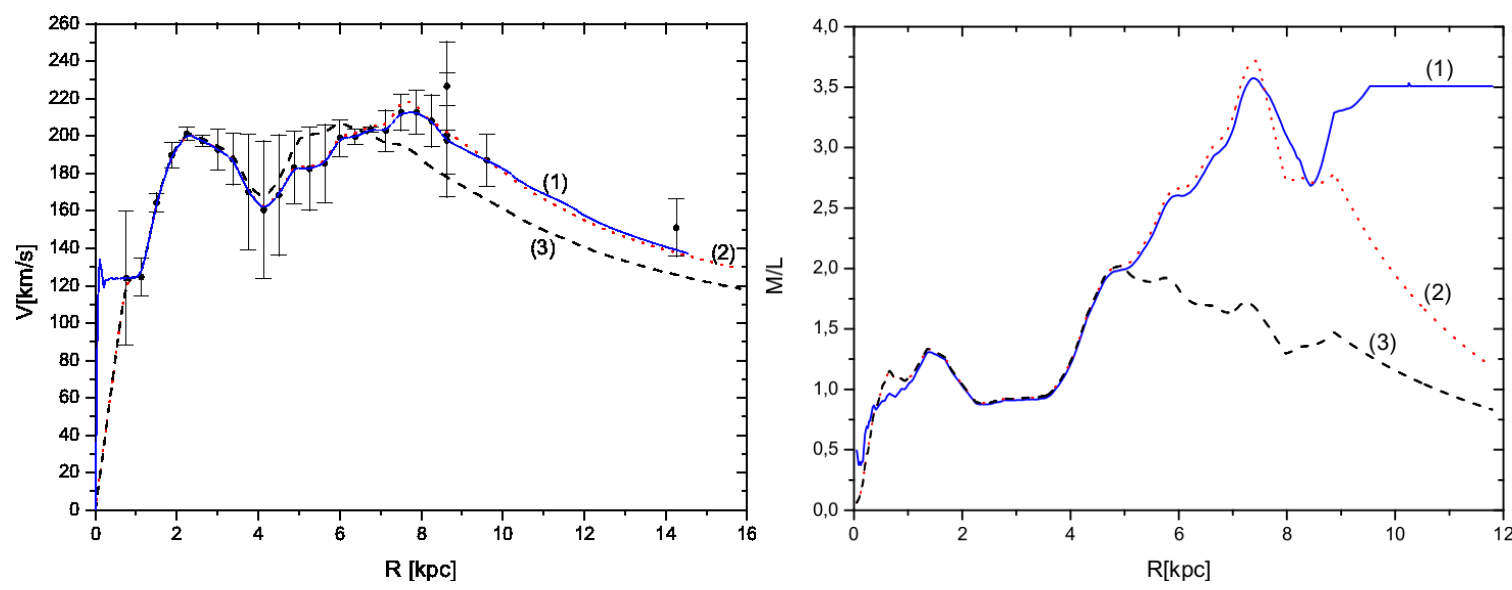

Figure 2: Rotation curves (left panel) and resulting mass-to-light ratio profiles (right panel) in disk model of galaxy NGC 253. The full circles with error bars in the left panel represent the rotation measurements from [14]. The solid line (labelled 1) - a rotation curve that fits in the rotation data within error bars (with some excess of mass density over the observed gas density). The dotted line (labelled 2) - an example of a small modification of rotation curve (1) for which the resulting mass-to-light ratio profile becomes decreasing (in disk model a small change in the rotation can largely influence the estimation of column mass density). The dashed line (labelled 3) denotes a rotation curve lowered at larger radii in such a way to account for some hypothetical dynamical contribution of the magnetic tension to the motion of gas, consistently with the magnetic field strength values observed in this galaxy. The corresponding mass-to-light ratio profile is significantly reduced and decreases with higher galactocentric distances, while at the same time the mass density remains consistent with the amount of visible gas at large radii. The figures were reconstructed based on our results published in [12].

same region [15]. In [16] we determined the maximum likelihood stellar and gaseous smooth outer rotation curves using a greater number of velocity tracers (the conditional distribution functions and smoothing algorithm is described therein). The curves are shown in figure 3 together with the position-velocity data used to obtain these curves. The difference between the two curves became more visible, and turned out to be increasing with the galacto-centric distance. This increase of the difference is what one should expect in magnetic field, because the density of mass drops off with the distance at large radii while the gravitational force weakens with the distance (thereby relatively enhancing the dynamical effect of magnetic tension on ionised gas).

In the Galactic plane vicinity we model the magnetic field by finding a solution of the stationary Navier-Stokes equation for an inviscid and pressureless medium. In this region we assume the vertical component of magnetic field to be vanishing to first order in the distance $Z$ from the galactic plane, while we keep the radial and the azimuthal components of that field, respectively, $B_{R}$ and $B_{\Phi}$. In this situation the equations in cylindrical cordinates $(R, \Phi, Z)$ reduce to

$$
V_{R} \frac{\partial V_{R}}{\partial R}=\frac{\delta v_{\Phi}^{2}}{R}-\frac{1}{4 \pi \rho} \frac{B_{\Phi}^{2}}{R}-\frac{1}{4 \pi \rho} B_{\Phi} \frac{\partial B_{\Phi}}{\partial R}, \quad V_{R} \frac{\partial\left(R V_{\Phi \mid G}\right)}{\partial R}=\frac{1}{4 \pi \rho} B_{R} \frac{\partial\left(R B_{\Phi}\right)}{\partial R} .
$$

We assume that all quantities depend solely on the radial distance $R$ from the axis of cylindrical symmetry. Here, $\rho$ is the volume density of gas corresponding to a column mass density $\sigma(R)$ according to equation $\rho(R, Z)=\frac{\sigma(R)}{4 h \cosh ^{2}\left(\frac{1}{2}(Z / h)\right)}$, where $h$ is a width-scale parameter. The $\sigma(R)$ can 


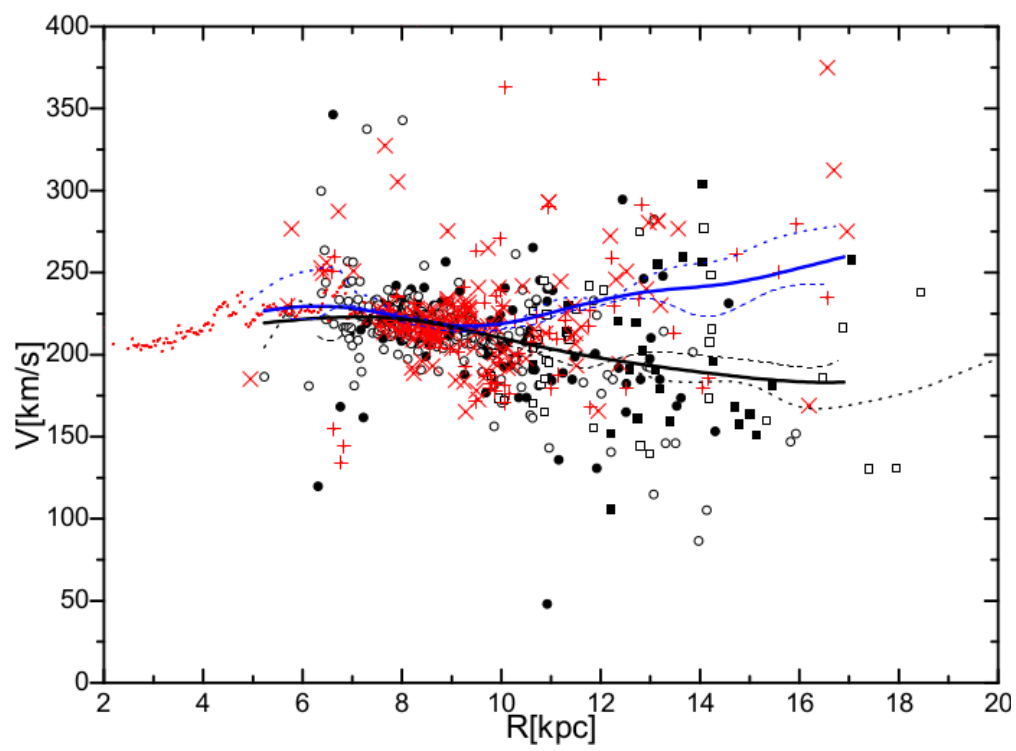

Figure 3: Maximum likelihood stellar and gaseous rotation curves we obtained for the Milky Way galaxy in [16] based on then available position-velocity data for stars and gas, assuming IAU Galactic constants $R_{o}=8.5 \mathrm{kpc}$ and $V_{o}=220 \mathrm{~km} / \mathrm{sec}$. The position-velocity points for star and gas tracers in the northern (N) and southern (S) side are shown for cepheids $\mathrm{N}$ (solid circles), cepheids $\mathrm{S}$ (open circles), carbon stars $\mathrm{S}$ (open squares), carbon stars $\mathrm{N}$ (solid squares), gaseous clouds $\mathrm{S}$ (stars) and gaseous clouds $\mathrm{N}$ (pluses) (error bars are not shown for clarity). The blue solid line is the gaseous rotation curve, the grey solid line is the stellar rotation curve. The dashed lines show the corresponding rotation curves when only objects above or below the Galactic plane are taken into account.

be reliably approximated by the column mass density of neutral hydrogen which we adopted from [19]. The quantity $\delta v_{\Phi}^{2}=V_{\Phi \mid G}^{2}-V_{\Phi \mid S}^{2}$ is the difference of squares of circular velocities of gas $V_{\Phi \mid G}$ and that of stars $V_{\Phi \mid S}$.

Suppose that $\delta v_{\Phi}^{2}$ can be entirely attributed to the interaction of the partially ionised gas with the large-scale magnetic field of the Galaxy. Then, we may predict a possible form and strength of the dominant part of the required field. The dominant field is likely to be axi-symmetric in agreement with the turbulent dynamo theory and observations [18]. To solve the above equations, we assume that in the disc plane vicinity $B_{R}=B(R) \sin \varepsilon$ and $B_{\Phi}=B(R) \eta \cos \varepsilon, \eta= \pm 1,|\varepsilon|<\frac{\pi}{2}$, where $B(R)$ is some positive function ( $\eta$ and a small perturbation parameter $\varepsilon$ set various directions of the field; we assume $\varepsilon \approx \frac{\pi}{12}$ after [20]). ${ }^{1}$ The residual rotation $\delta v_{\Phi}^{2}$ is insensitive to the direction of the total magnetic field and to the sign of $B_{\Phi}$. As we explained in [16], the magnetic field ansatz may be regarded as consisting both of a large-scale purely azimuthal field and a correction that mimics turbulent field. The magnitudes of turbulent fields in the Galaxy may be comparable or even exceeding the magnitudes of large-scale fields. Given $V_{\Phi \mid S}, V_{\Phi \mid G}$ and $\rho$, the above equations can be reduced to a single non-linear ordinary equation for $B(R)$ that can be solved numerically. The result is shown in figure 4 .

\footnotetext{
${ }^{1}$ The divergence of magnetic field is $R^{-1} \partial_{R}(R B(R)) \sin \varepsilon$. The divergence can be disregarded only when $\varepsilon$ is small enough or/and when $B(r)$ is close to a function const $\cdot R^{-1}$. However, the purpose of the model is to approximately determine the leading structure of the horizontal magnetic field, therefore we may allow for a small violation of the
} 

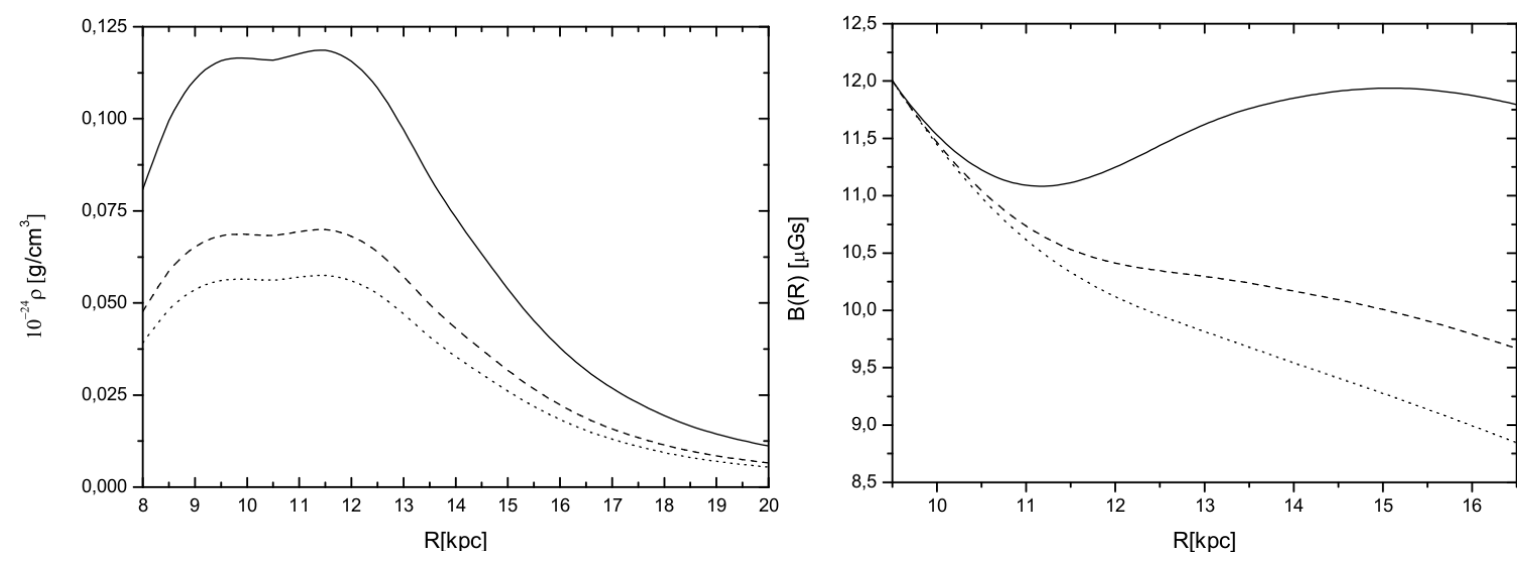

Figure 4: Left panel: volume density of the gas in the Milky Way Galaxy at $Z=0$ for various width-scale parameter $h: 2 \mathrm{kpc}$ (solid line), $4 \mathrm{kpc}$ (dashed line) and $6 \mathrm{kpc}$ (dotted line). Right panel: the corresponding magnitude of the large-scale magnetic field that accounts for the observed difference between the stellar and gaseous rotation curve of the Milky Way Galaxy.

\section{Conclusions}

Magnetic fields influence the motion of gas in spiral galaxies, modifying their rotation curves. For the magnetic effect to occur, the gas must be at least partially ionised, and the effect enhances for more rarefied gas, especially in the galaxy outskirts or at higher altitudes above the galactic plane.

In the disk model framework, applicable to flattened mass distributions, there is a high sensitivity of the modelled mass-to-light ratio profile in the outer galactic regions to small perturbations of the rotation curve (even within the rotation curve measurement uncertainties). In this region, as we have seen on the example of galaxy NGC 253, the inclusion of magnetic fields allowed to significantly reduce the mass-to-light ratio and even invert its tendency of growth. In view of the observation that the difference between the gaseous and stellar rotation curves may be increasing for outermost radii (as it seems to be the case for our Galaxy) this may have implications for the missing mass problem and the estimations of the amount of non-baryonic dark matter in spiral galaxies.

The results we have presented show that magnetic fields cannot be neglected in modelling galactic rotation curves. We are not sure if the real magnetic fields in NGC 891, NGC 253 or in the Galaxy are characterized by a similar dependence on the galactocentric distance to cause the effects we postulated. At least, the overall field magnitudes we have obtained are of the right order, consistent with measurements.

\section{References}

[1] E. Battaner, J. L. Garrido, M. Membrado and E. Florido, Magnetic fields as an alternative explanation for the rotation curves of spiral galaxies, Nature 360 (1992) 652.

divergence freedom by the partial field, assuming that it is cancelled by corrections to the total field. 
[2] E. Battaner and E. Florido, Are rotation curves in NGC 6946 and the Milky Way magnetically supported?, Astronomische Nachrichten 328 (2007) 92 [astro-ph / 0503657 ].

[3] B. Ruiz-Granados, E. Battaner, J. Calvo, E. Florido and J. A. Rubiño-Martín, Dark Matter, Magnetic Fields, and the Rotation Curve of the Milky Way, Astrophysical Journal, Letters 755 (2012) L23.

[4] F. J. Sánchez-Salcedo and A. Santillán, Magnetic fields: impact on the rotation curve of the Galaxy, Monthly Notices of the Royal Astronomical Observatory 433 (2013) 2172 [1302 . 7253 ].

[5] D. Elstner, R. Beck and O. Gressel, Do magnetic fields influence gas rotation in galaxies?, Astronomy and Astrophysics 568 (2014) A104 [1407.6998].

[6] J. Jałocha, Ł. Bratek, J. Pȩkala and M. Kutschera, The role of large-scale magnetic fields in galaxy NGC 891: can magnetic fields help to reduce the local mass-to-light ratio in the galactic outskirts?, Monthly Notices of the Royal Astronomical Observatory 421 (2012) 2155 [1111.6417].

[7] F. Fraternali, R. Sancisi and P. Kamphuis, A tale of two galaxies: light and mass in NGC 891 and NGC 7814, Astronomy and Astrophysics 531 (2011) A64 [1105.3867].

[8] T. Oosterloo, F. Fraternali and R. Sancisi, The Cold Gaseous Halo of NGC 891, Astronomical Journal 134 (2007) 1019 [0705.4034].

[9] R. J. Allen and S. Sukumar, NGC 891: a Summary of Observations, in The Interstellar Disk-Halo Connection in Galaxies, H. Bloemen, ed., vol. 144 of IAU Symposium, p. 287, Jan, 1991.

[10] M. Dumke, M. Krause, R. Wielebinski and U. Klein, Polarized radio emission at $2.8 \mathrm{~cm}$ from a selected sample of edge-on galaxies., Astronomy and Astrophysics 302 (1995) 691.

[11] E. Hummel, M. Dahlem, J. M. van der Hulst and S. Sukumar, The large-scale radio continuum structure of the edge-on spiral galaxy NGC 891., Astronomy and Astrophysics 246 (1991) 10.

[12] J. Jałocha, Ł. Bratek, J. Pȩkala and M. Kutschera, A possible influence of magnetic fields on the rotation of gas in NGC 253, Monthly Notices of the Royal Astronomical Observatory 427 (2012) 393 [1210.3082].

[13] V. Heesen, M. Krause, R. Beck and R.-J. Dettmar, The Radio Halo of the Starburst Galaxy NGC 253, in The Magnetized Plasma in Galaxy Evolution, K. T. Chyzy, K. Otmianowska-Mazur, M. Soida and R.-J. Dettmar, eds., pp. 156-161, June, 2005.

[14] J. Hlavacek-Larrondo, C. Carignan, O. Daigle, M. M. de Denus-Baillargeon, M. Marcelin, B. Epinat

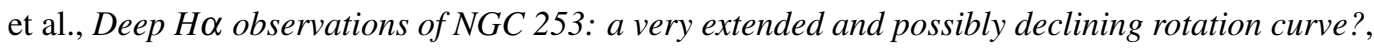
Monthly Notices of the Royal Astronomical Observatory 411 (2011) 71 [1009.1177].

[15] F. Pont, D. Queloz, P. Bratschi and M. Mayor, Rotation of the outer disc from classical cepheids., Astronomy and Astrophysics 318 (1997) 416.

[16] J. Jałocha, Ł. Bratek, J. Pȩkala, S. Sikora and M. Kutschera, Magnetic Flux Density from the Relative Circular Motion of Stars and Partially Ionized Gas in the Galaxy Mid-plane Vicinity, Astrophysical Journal 833 (2016) 174 [1610.07544].

[17] D. P. Clemens, Massachusetts-Stony Brook Galactic plane CO survey: the galactic disk rotation curve., Astrophysical Journal 295 (1985) 422.

[18] J. P. Vallee, Reversing the Axisymmetric $(m=0)$ Magnetic Fields in the Milky Way, Astrophysical Journal 366 (1991) 450.

[19] H. Nakanishi and Y. Sofue, Three-dimensional distribution of the ISM in the Milky Way galaxy. III. The total neutral gas disk, Publications of the Astronomical Society of Japan 68 (2016) 5 [1511.08877]. 
[20] B. Ruiz-Granados, E. Battaner, J. Calvo, E. Florido and J. A. Rubiño-Martín, Dark Matter, Magnetic Fields, and the Rotation Curve of the Milky Way, Astrophysical Journal, Letters 755 (2012) L23.

[21] P. M. W. Kalberla and J. Kerp, The Hi Distribution of the Milky Way, Annual Review of Astronomy and Astrophysics 47 (2009) 27. 